\title{
ANSIEDAD ANTE LA AGRESIÓN Y ANTE EL FRACASO ESCOLAR Y SANCIONES DISCIPLINARIAS Y RASGOS PERFECCIONISTAS
}

\author{
José Daniel Alvarez Teruel \\ Universidad de Alicante \\ josedaniel.alvarez@ua.es \\ Ma Pilar Aparicio Flores \\ Universidad de Alicante \\ pilar.aparicio@ua.es \\ Carlos Edisson Jimenez Ayala \\ Universidad Central del Ecuador \\ cjimeneza@hotmail.com \\ Nancy Cargua García \\ Universidad Central del Ecuador \\ ncarguag@yahoo.es \\ José Manuel García-Fernández \\ Universidad de Alicante \\ josemagf@ua.es
}

Recepción Artículo: 27 octubre 2021

Admisión Evaluación: 27 octubre 2021

Informe Evaluador 1: 28 octubre 2021

Informe Evaluador 2: 29 octubre 2021

Aprobación Publicación: 30 octubre 2021

\section{RESUMEN}

La ansiedad es uno de los trastornos emocionales con mayor relevancia en la población, teniendo en cuenta la excesiva exigencia marcada por la sociedad actual. Esta exigencia desmedida marcada, en ocasiones, por un rasgo de la personalidad perfeccionista, todavía tiende a agravar más si cabe los estados ansiosos y estresantes del individuo. En este sentido, es importante tener presente la etapa estudiantil la cual se enfrenta continuamente a situaciones estresantes. Por ello, es conveniente observar cuál es el vínculo entre las variables perfeccionistas, regidas por el Perfeccionismo Socialmente Prescrito (PSP) y el Perfeccionismo Auto-Orientado (PAO) y la Ansiedad Escolar, compuesta, entre otras, por la Ansiedad ante la agresión y la Ansiedad ante el fracaso escolar y las sanciones disciplinarias. De ahí que el objetivo del presente estudio sea observar si existen diferencias estadísticamente significativas entre el Factor I y II del Inventario de Ansiedad Escolar (IAES) y el PSP y PA0, así como determinar la probabilidad de presentar altas puntuaciones en ambos factores de la Ansiedad Escolar en función del PSP y el PA0. Para ello, se reclutó una muestra de 1588 estudiantes ecuatorianos y se utilizaron medidas como el IAES y el Child and Adolescent Perfectionism Scale (CAPS). Los resultados arrojaron diferencias estadísticamente significativas para el Factor I y II del IAES tanto en PSP como en PAO, siendo estas de baja y moderada magnitud. Asimismo, se observa que a medida que aumenta el PSP y el PAO aumenta la posibilidad de pre- 


\section{ANSIEDAD ANTE LA AGRESIÓN Y ANTE EL FRACASO ESCOLAR Y SANCIONES DISCIPLINARIAS Y RASGOS PERFECCIONISTAS}

sentar elevadas puntuaciones en Ansiedad ante la agresión y en Ansiedad ante el fracaso escolar y las sanciones disciplinarias. En conclusión, los hallazgos muestran que es necesario incrementar el número de estudios en variables perfeccionistas en el país de Ecuador, teniendo en cuenta su vínculo con la Ansiedad Escolar y lo que ello conlleva.

Palabras clave: ansiedad escolar; perfeccionismo; perfeccionismo socialmente prescrito; perfeccionismo auto-orientado

\section{ABSTRACT}

Aggression anxiety and to failure and school punishment and perfectionist traits. Anxiety is one of the most relevant emotional disorders in the population, taking into account the excessive demand set by today's society. This excessive demand, sometimes marked by a perfectionist personality trait, tends to further aggravate the anxious and stressful states of the individual. In this sense, it is important to keep in mind the student stage, which continually faces stressful situations. Therefore, it is convenient to observe the link between the perfectionist variables, governed by Socially Prescribed Perfectionism (SPP) and Self-Oriented Perfectionism (SOP) and School Anxiety, composed, among others, by Anxiety in the face of aggression and Anxiety about school failure and disciplinary sanctions. Hence, the objective of the present study is to observe if there are statistically significant differences between Factor I and II of the School Anxiety Inventory (IAES) and the SPP and SOP, as well as to determine the probability of presenting high scores in both Anxiety factors. School based on the SPP and the SOP. For this, a sample of 1588 Ecuadorian students was recruited and measures such as the IAES and the Child and Adolescent Perfectionism Scale (CAPS) were used. The results yielded statistically significant differences for IAES Factor I and II in both SPP and SOP, these being of low and moderate magnitude. Likewise, it is observed that as the SPP and the SOP increase, the possibility of presenting high scores in Anxiety in the face of aggression and in Anxiety in the face of school failure and disciplinary sanctions increases. In conclusion, the findings show that it is necessary to increase the number of studies on perfectionist variables in the country of Ecuador, taking into account its link with School Anxiety and what this entails.

Keywords: school anxiety; perfectionism; socially prescribed perfectionism; self-oriented perfectionism

\section{INTRODUCCIÓN}

La ansiedad se considera una de las problemáticas emocionales con mayor relevancia y dominancia en la población (Baxter et al., 2013; Kessler et al., 2007; Méndez, et al. 2008; Steel et al., 2014), teniendo en cuenta que la excesiva exigencia y los cambios continuados y a pasos agigantados que desencadena la sociedad actual provoca desórdenes de carácter ansioso tanto en población adulta (Demyttenaere, 2004; Kessler et al., 2007) como en la infancia y/o adolescencia (In-Albon y Schneider, 2007). Sin embargo, poco se conoce sobre la ansiedad escolar en edades comprendidas entre 12 y 18 años en el país de Ecuador.

Pese a que la mayoría de las personas considera que este tipo de desórdenes suelen ocurrir en la edad adulta, Io cierto es que muchos trastornos ansiosos tienen su origen alrededor de los once años de edad, siendo uno de los problemas emocionales con más trascendencia en el desarrollo de los infantes (Beesdo et al., 2009; Kessler et al., 2007), el cual suele alargarse en el tiempo (Dadds et al., 1999).

Es, por tanto, importante determinar estudios que examinen los trastornos ansiosos en la población infantojuvenil ecuatoriana, teniendo en cuenta que, pese a que tanto la ansiedad como los miedos son reacciones habituales en la edad infantil (Méndez et al., 2003), en ocasiones, puede llegar a persistir e incluso a aumentar, llegando a transformarse en comportamientos emocionalmente problemáticos y desadaptativos, haciendo que el infante, y posteriormente, el adulto, no se ajuste adecuadamente al entorno (Espada, et al., 2012; Van Ameringen et al., 2003).

En la actualidad, la población estudiantil se enfrenta continuamente a situaciones de gran presión, como por ejemplo la adaptación a nuevos profesores, compañeros y a toda la diversidad existente en el centro, las evaluaciones a las que se someten, y la competitividad entre iguales. Ahí entran en juego algunas dimensiones de la 
ansiedad escolar tales como la Ansiedad ante la agresión y/o la Ansiedad ante el fracaso escolar y las sanciones disciplinarias, las cuales serán objeto de estudio. Estas situaciones hacen que muchos niños y adolescentes afirmen que la escuela es uno de los contextos de mayor base ansiógena y estresante a los que se someten (Sung et al., 2006).

Al mismo tiempo, la excesiva presión, muchas veces marcadas por la sociedad, regido por un Perfeccionismo Socialmente Prescrito (PSP), y en otras muchas ocasiones por el propio individuo, basado en un Perfeccionismo Autorientado (PA0), puede ocasionar un rasgo de la personalidad perfeccionista. El Perfeccionismo, por su parte, es un rasgo multidimensional que afecta negativamente a la vida de las personas que lo padecen (Babapour et al., 2015) debido al nerviosismo ante cualquier situación que no sea perfecta y a su vínculo con psicopatologías como el agotamiento y desgaste mental (Mahmoodi-Shahrebabaki, 2017), malestar personal (Aparicio-Flores et al., 2021), la ansiedad social (Esteve-Faubel et al., 2020), agresividad (Vicent, Inglés, Gonzálvez et al., 2019) y dificultades interpersonales (Aparicio-Flores et al., 2020) entre otras.

En este sentido, un rasgo perfeccionista podría vincularse a un aumento de ansiedad escolar. De ahí que sea importante observar el nexo de unión entre el Perfeccionismo y la Ansiedad ante la agresión y la Ansiedad ante el fracaso escolar y las sanciones disciplinarias, factores de la Ansiedad escolar.

\section{OBJETIVOS DE LA INVESTIGACIÓN}

El objetivo del estudio es observar el vínculo entre la Ansiedad ante la agresión y la Ansiedad ante el fracaso escolar y las sanciones disciplinarias y el PSP y el PAO en estudiantes ecuatorianos entre 12 y 18 años. Concretamente, los objetivos específicos del estudio se concretan en: a) observar si existen diferencias estadísticamente significativas en la Ansiedad ante la agresión y la Ansiedad ante el fracaso escolar y las sanciones disciplinarias y el PSP y el PAO; y b) analizar la probabilidad de aumento de la Ansiedad ante la agresión y la Ansiedad ante el fracaso escolar y las sanciones disciplinarias sobre el aumento del PSP y PAO en adolescentes ecuatorianos.

\section{MUESTRA Y/O PARTICIPANTES}

La muestra se conformó, bajo un muestreo aleatorio por conglomerados, por un total de 1725 estudiantes ecuatorianos de la ciudad de Quito con edades comprendidas entre 12 y 18 años. La muestra total se conformó por 1588 participantes ( $M=14.82$; D.E.= 1.86) tras eliminar 137 estudiantes por errores u omisiones en sus respuestas 0 por no tener el consentimiento de los padres. Los estudiantes cursaban desde Octavo hasta $3^{0}$ de Bachillerato, de los cuales el $60.9 \%$ son varones.

\section{METODOLOGÍA Y/O INSTRUMENTOS UTILIZADOS}

\section{Instrumentos}

Inventario de Ansiedad Escolar (IAES; García-Fernández et al., 2011): El IAES se trata de una escala de medida para la evaluación de la Ansiedad escolar en adolescentes entre 12 y 18 años. El IAES se compone de una tabla de doble entrada. El eje horizontal de la escala se compone de 25 ítems, con 5 opciones de respuesta ( $0=$ nunca, $4=$ siempre), que evalúan situaciones que suelen darse en la escuela. Estas situaciones se miden a través de 4 dimensiones: F1. Ansiedad ante el fracaso y castigo escolar; F2. Ansiedad ante la evaluación social. F3. Ansiedad ante la agresión; F4. Ansiedad ante la evaluación escolar. Por otra parte, el eje vertical se compone de 19 ítems que hacen referencia a la Ansiedad cognitiva (9 ítems), Ansiedad conductual (5 ítems) y Ansiedad psicofisiológica (5 ítems).

Los coeficientes de consistencia interna fueron aceptables para todos los factores ( = entre .88 y .93), así como para los tres tipos de ansiedad ( $=.86, .82$ y .86 respectivamente). Del mismo modo, también fue aceptable su fiabilidad test-retest, en un intervalo de dos semanas ( = entre .78 y .84 para el eje horizontal; $y=$ entre $.74 \mathrm{y}$ .77 para los tres tipos de ansiedad del eje vertical). 


\section{ANSIEDAD ANTE LA AGRESIÓN Y ANTE EL FRACASO ESCOLAR Y SANCIONES DISCIPLINARIAS Y RASGOS PERFECCIONISTAS}

Child and Adolescent Perfectionism Scale (CAPS; Flett et al., 2016; Vicent, Inglés, Sanmartín et al., 2019): La CAPS es una escala de autoinforme, con 5 opciones de respuesta (1= Falso; No es del todo cierto para $m i ; 5=$ Muy cierto de mi), que se compone de 22 ítems que evalúan el Perfeccionismo en población infanto-juvenil. La versión original se compone de dos dimensiones: F1. PSP y F2. PAO. Su validación original obtuvo adecuados índices de fiabilidad ( $=.81$, para PAO, y $=.85$ para PSP), así como también lo hizo la versión española tanto para PSP como para PA0-críticas y PA0-esfuerzos ( $=.79, .77$ y .76, respectivamente; Vicent, Inglés, Sanmartín et al., 2019) y su versión ecuatoriana (Vicent et al., 2020).

\section{Procedimiento}

Se llevó a cabo una entrevista con el equipo directivo con el objetivo de solicitar permiso y colaboración para la investigación. Posteriormente, se informó a los padres y/o tutores de los participantes y se les pidió el consentimiento informado. Seguidamente, tras recibir el consentimiento informado de los participantes del estudio, se administraron las escalas para analizar los objetivos propuestos en la investigación. Los cuestionarios fueron explicados al inicio de su administración, su proceso fue voluntario y anónimo, y se contó con la presencia de uno de los investigadores y la ayuda del profesorado.

\section{Análisis de datos}

Los análisis de datos para medir la significatividad de las diferencias de las distintas dimensiones de la Ansiedad escolar y el Perfeccionismo se realizaron a través del índice $d$. Estos índices fueron analizados bajo los criterios propuestos por Cohen (1988), el cual estima que un tamaño del efecto pequeño se da entre .20 y .49, un tamaño moderado entre .50 y .79, y un tamaño grande en puntuaciones más elevadas que .80 .

La probabilidad de predicción de los factores de Perfeccionismo sobre los factores de la Ansiedad escolar se analizó mediante regresión logística, con el procedimiento de pasos hacia adelante basado en el estadístico de Wald. Esta probabilidad es estimada mediante el estadístico odd ratio $(O R)$, siguiendo la siguiente interpretación: si la $O R$ es $>1$, ej.: 2, por cada vez que se dé esa casuística en presencia de la variable independiente, se dará 2 veces si esta variable está presente. Si la $O R$ es $<1$, ej. 0.5 , la posibilidad de que se dé la casuística en ausencia de la variable independiente será 0.5 veces $<$ que en su presencia.

Se tuvieron en cuenta: la $R^{2}$ de Nagelkerke, que informa del \% de varianza explicada por el modelo (Nagelkerke, 1991), y el \% de casos clasificados correctamente por el modelo. Asimismo, la variable dependiente fue dicotomizada en bajas puntuaciones con un centil $<25$ y en altas puntuaciones con un centil $>75$.

\section{RESULTADOS ALCANZADOS}

\section{Diferencias en PSP y PAO en adolescentes con altas y bajas puntuaciones en el Factor I del IAES}

La Tabla 1 y Figura 1 muestran las medias y diferencias estadísticamente significativas observadas en Perfeccionismo desde adolescentes con elevadas y bajas puntuaciones en Ansiedad ante la agresión (Factor I del IAES).

Los resultados hallados arrojan diferencias estadísticamente significativas tanto en PSP ( $d=.46$ ) como en PAO $(d=.44)$ con una baja magnitud en ambos factores del Perfeccionismo.

Se observa que Ios alumnos con elevadas puntuaciones en el Factor I del IAES puntúan significativamente más elevado tanto en PSP como en PAO que aquellos estudiantes con bajas puntuaciones en Ansiedad ante la agresión. 
Tabla 1Diferencias en PSP y PAO en adolescentes ecuatorianos con altas y bajas puntuaciones en el Factor I del IAES

\begin{tabular}{|c|c|c|c|c|c|c|c|c|c|c|}
\hline Variable & \multicolumn{2}{|c|}{$\begin{array}{l}\text { Prueba } \\
\text { Levene }\end{array}$} & \multicolumn{2}{|c|}{$\begin{array}{c}\text { Altas } \\
\text { puntuaciones }\end{array}$} & \multicolumn{2}{|c|}{$\begin{array}{c}\text { Bajas } \\
\text { puntuaciones }\end{array}$} & \multicolumn{4}{|c|}{$\begin{array}{c}\text { Significación estadística y } \\
\text { magnitud diferencias }\end{array}$} \\
\hline & $F$ & $p$ & $M$ & $D E$ & $M$ & $D E$ & $t$ & g.l. & $p$ & $d$ \\
\hline PSP & 5.50 & .019 & 34.19 & 6.27 & 31.12 & 7.09 & 6.44 & 739.82 & $<.001$ & .46 \\
\hline PAO & 2.32 & .127 & 40.73 & 6.24 & 37.90 & 6.68 & 6.23 & 808 & $<.001$ & .44 \\
\hline
\end{tabular}

Figura 1. Diferencias en PSP y PAO en adolescentes con altas y bajas puntuaciones en el Factor I del IAES. * $=$ $p<.05,{ }^{* *} p<.01,{ }^{* * *} p<.001$

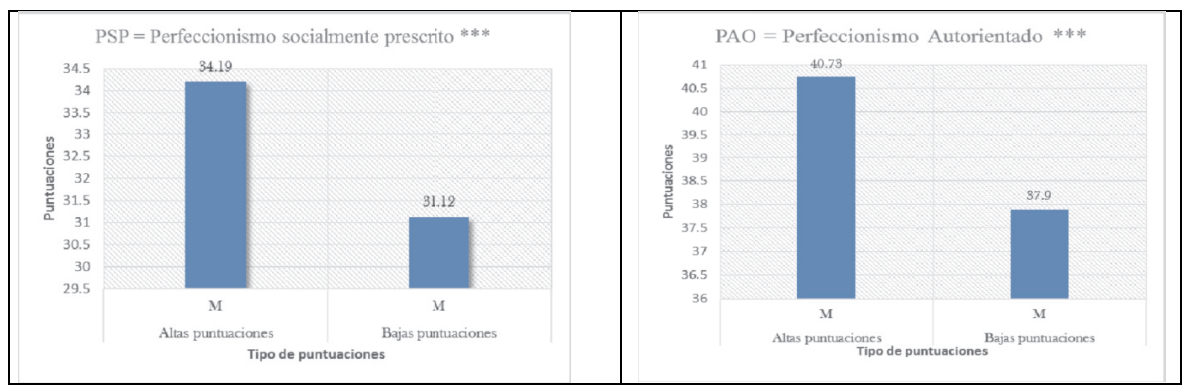

\section{Regresión logística binaria para la probabilidad de presentar alta puntuación en el Factor I del IAES en función del PSP y PAO}

La Tabla 2 muestra los modelos resultantes de la regresión logística binaria para la probabilidad de presentar altas puntuaciones en Ansiedad ante la agresión en función de las variables de Perfeccionismo.

Los resultados hallados permiten hacer una estimación correcta del $60.5 \%$ de los casos ( $2=41.24 ; p=<.05$ ) para el PSP y del $59.5 \%$ de los casos ( ${ }^{2}=37.99 ; p=<.05$ ) para el PAO. El valor de ajuste de los modelos ( ${ }^{2}$ Nagelkerke) fue de .07 para el PSP y del .06 para el PAO.

Los valores de la odd ratio $(O R)$ fueron > 1 señalando que a medida que aumenta la puntuación en PSP y PAO aumenta la posibilidad de presentar elevadas puntuaciones en Ansiedad ante la agresión. Los valores obtenidos fueron 1.07 tanto para PSP como PAO. 
Tabla 2 Regresión logística binaria para la probabilidad de presentar elevadas puntuaciones en el Factor I del IAES en función de PSP y PAO

\begin{tabular}{|c|c|c|c|c|c|c|c|c|c|}
\hline $\begin{array}{l}\text { Variabl } \\
\mathrm{e}\end{array}$ & & $\chi^{2}$ & $\mathrm{R}^{2}$ & B & E.T. & Wald & $p$ & $O R$ & $\begin{array}{r}\text { I.C. } \\
95 \% \\
\end{array}$ \\
\hline \multirow[t]{4}{*}{ PAO } & Clasificados & 37. & 0 & & & 35.4 & $<.00$ & & \multirow{4}{*}{$\begin{array}{r}1.04- \\
1.09\end{array}$} \\
\hline & $\begin{array}{l}\text { correctament } \\
\text { e: } 59.5 \%\end{array}$ & 99 & 6 & .06 & .01 & $\begin{array}{r}75.4 \\
7\end{array}$ & & $\begin{array}{r}1.0 \\
7\end{array}$ & \\
\hline & Constante & & & - & .45 & 30.1 & $<.00$ & .08 & \\
\hline & & & & 2.50 & & 7 & 1 & & \\
\hline \multirow{3}{*}{ PSP } & Clasificados & 41. & .0 & 06 & 01 & 38.6 & $<.00$ & 1.0 & \multirow{3}{*}{$\begin{array}{r}1.04- \\
1.09\end{array}$} \\
\hline & e: $60.5 \%$ & 24 & 7 & .00 & .01 & 5 & & 7 & \\
\hline & Constante & & & 2.05 & .36 & $\begin{array}{r}31.4 \\
5\end{array}$ & $\begin{array}{l}<.00 \\
1\end{array}$ & .12 & \\
\hline
\end{tabular}

Nota: $\mathrm{PAO}=$ Perfeccionismo Autorientado, $\mathrm{PSP}=$ Perfeccionismo Socialmente Prescrito, $\chi^{2}=$ Chi cuadrado; $\mathrm{R}^{2}=$ Cuadrado de Nagelkerke; $\mathrm{B}=$ Coeficiente de regresión; E.T.= Error estándar; Wald $=$ Prueba de Wald; $p=$ Probabilidad; $O R=$ Odd ratio; I.C.= Intervalo de confianza al 95\%.

\section{Diferencias en PSP y PAO en adolescentes con altas y bajas puntuaciones en Ansiedad ante el fracaso escolar y sanciones disciplinarias}

La Tabla 3 y Figura 2 muestran las medias y diferencias estadísticamente significativas en PSP y PAO en adolescentes con altas y bajas puntuaciones en Ansiedad por fracaso escolar y sanciones disciplinarios (Factor II del IAES).

Los hallazgos muestran diferencias significativas de elevada magnitud para el PSP ( $d=.53)$ y de baja magnitud para el PAO ( $d=.41)$, sugiriendo que los adolescentes con elevadas puntuaciones en Ansiedad ante el fracaso escolar y sanciones disciplinarias puntúa significativamente más alto en ambas facetas del perfeccionismo que sus iguales con bajas puntuaciones en el Factor II del IAES.

Tabla 3Diferencias en PSP y PAO en adolescentes con altas y bajas puntuaciones en la Ansiedad ante el fracaso escolar y sanciones disciplinarias.

\begin{tabular}{|c|c|c|c|c|c|c|c|c|c|c|}
\hline \multirow[t]{2}{*}{$\begin{array}{l}\text { Variabl } \\
\mathrm{e}\end{array}$} & \multicolumn{2}{|c|}{$\begin{array}{l}\text { Prueba } \\
\text { Levene }\end{array}$} & \multicolumn{2}{|c|}{$\begin{array}{c}\text { Altas } \\
\text { puntuaciones }\end{array}$} & \multicolumn{2}{|c|}{$\begin{array}{c}\text { Bajas } \\
\text { puntuaciones }\end{array}$} & \multicolumn{4}{|c|}{$\begin{array}{c}\text { Significación estadística y } \\
\text { magnitud diferencias }\end{array}$} \\
\hline & $F$ & $p$ & $M$ & $D E$ & $M$ & $D E$ & $t$ & g.l. & $p$ & $d$ \\
\hline PSP & 6.0 & .01 & 34.7 & 6.4 & 31.1 & 7.2 & 7.3 & 701.4 & $<.00$ & .5 \\
\hline & 2 & 4 & 7 & 2 & 4 & 7 & 2 & 6 & 1 & 3 \\
\hline PAO & .71 & $\begin{array}{r}.39 \\
9\end{array}$ & $\begin{array}{r}40.7 \\
8\end{array}$ & $\begin{array}{r}6.3 \\
5\end{array}$ & $\begin{array}{r}38.1 \\
1\end{array}$ & $\begin{array}{r}6.6 \\
9\end{array}$ & $\begin{array}{r}5.7 \\
1\end{array}$ & 787 & $\begin{array}{r}<.00 \\
1\end{array}$ & $\begin{array}{l}.4 \\
1\end{array}$ \\
\hline
\end{tabular}


Figura 2. Diferencias en PSP y PAO en adolescentes con altas y bajas puntuaciones en el Factor II del IAES. *= $p<.05,{ }^{* \star} p<.01,{ }^{* \star *} p<.001$

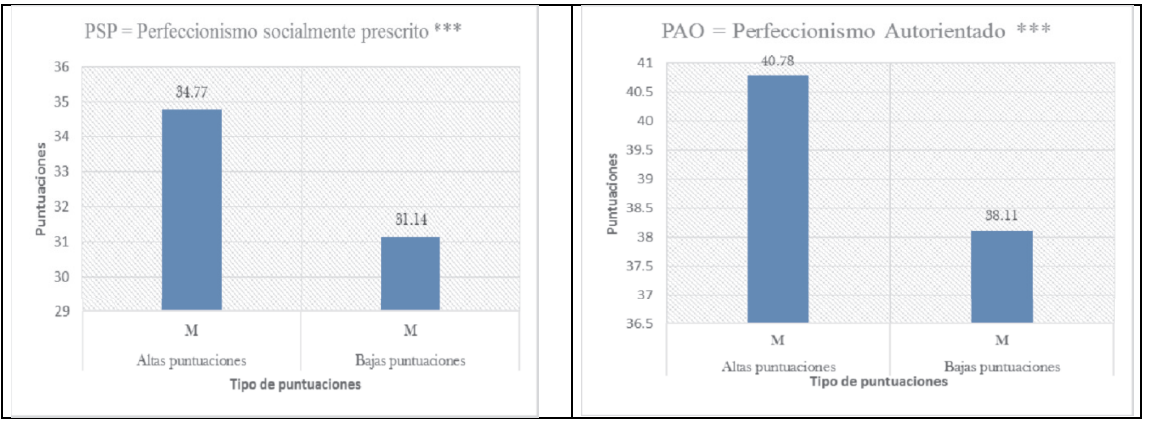

\section{Regresión logística binaria para la probabilidad de presentar alta puntuación en el Factor II del IAES en función de PSP y PAO}

La Tabla 4 muestra los modelos resultantes de la regresión logística binaria sobre la probabilidad de presentar elevadas puntuaciones de Ansiedad ante el fracaso escolar y sanciones disciplinarias.

Los resultados permiten realizar una estimación correcta del $62.4 \%$ de los casos $\left({ }^{2}=53.01 ; p=<.05\right)$ para el PSP y del $60.7 \%$ de los casos ( ${ }^{2}=32.10 ; p=<.05$ ) para el PAO. Del mismo modo, el valor de ajuste de los modelos ( $R^{2}$ Nagelkerke) comprende entre .05 para el PAO y .09 para el PSP.

Los valores de la odd ratio $(O R)$ fueron $>1$ sugiriendo que conforme aumenta la puntuación en PSP y PAO aumenta la probabilidad de presentar elevadas puntuaciones en el Factor II del IAES, con valores de 1.08 para el PSP y 1.06 para el PAO.

Tabla 4Regresión logística binaria para la probabilidad de presentar alta puntuación en el Factor II del IAES en función de PSP y PAO

\begin{tabular}{|c|c|c|c|c|c|c|c|c|c|}
\hline $\begin{array}{l}\text { Variabl } \\
\mathrm{e}\end{array}$ & & $\chi^{2}$ & $\mathrm{R}^{2}$ & B & E.T. & Wald & $p$ & $O R$ & $\begin{array}{r}\text { I.C. } \\
95 \%\end{array}$ \\
\hline \multirow[t]{2}{*}{$\mathrm{PAO}$} & $\begin{array}{l}\text { Clasificados } \\
\text { correctamente: } \\
60.7 \%\end{array}$ & 32.10 & .05 & .06 & .01 & $\begin{array}{r}30.2 \\
4\end{array}$ & $\begin{array}{l}<.00 \\
1\end{array}$ & $\begin{array}{r}1.0 \\
6\end{array}$ & \multirow[t]{2}{*}{$\begin{array}{r}1.04- \\
1.08\end{array}$} \\
\hline & Constante & & & $2.2 \overline{6}^{-}$ & .45 & $\begin{array}{r}24.5 \\
3\end{array}$ & $\begin{array}{l}<.00 \\
1\end{array}$ & .10 & \\
\hline \multirow[t]{2}{*}{ PSP } & $\begin{array}{l}\text { Clasificados } \\
\text { correctamente: } \\
62.4 \%\end{array}$ & 53.01 & .09 & .07 & .01 & $\begin{array}{r}48.8 \\
9\end{array}$ & $\begin{array}{l}<.00 \\
1\end{array}$ & $\begin{array}{r}1.0 \\
8\end{array}$ & \multirow[t]{2}{*}{$\begin{array}{r}1.05- \\
1.10\end{array}$} \\
\hline & Constante & & & $2.31^{-}$ & .37 & $\begin{array}{r}39.2 \\
3 \\
\end{array}$ & $\begin{array}{l}<.00 \\
1\end{array}$ & .09 & \\
\hline
\end{tabular}

Nota: $\mathrm{PAO}=$ Perfeccionismo autorientado, $\mathrm{PSP}=$ Perfeccionismo socialmente prescrito, $\chi^{2}=$ Chi cuadrado; $\mathrm{R}^{2}=$ Cuadrado de Nagelkerke; $\mathrm{B}=$ Coeficiente de regresión; E.T.= Error estándar; Wald $=$ Prueba de Wald; $p=$ Probabilidad; $O R=$ Odd ratio; I.C.= Intervalo de confianza al 95\%. 


\section{ANSIEDAD ANTE LA AGRESIÓN Y ANTE EL FRACASO ESCOLAR Y SANCIONES DISCIPLINARIAS Y RASGOS}

PERFECCIONISTAS

\section{DISCUSIÓN}

El objetivo del estudio era observar si existen diferencias estadísticamente significativas en la Ansiedad ante la agresión y la Ansiedad ante el fracaso escolar y sanciones disciplinarias y el PSP y el PAO, así como observar si aumenta la Ansiedad ante la agresión y la Ansiedad ante el fracaso escolar y las sanciones disciplinarias a medida que aumenta el PSP y el PAO en alumnado ecuatoriano entre 12 y 18 años.

Con respecto al primer objetivo, las diferencias fueron estadísticamente significativas en ambos casos tanto en PSP como en PA0. En este sentido, Ios adolescentes con elevadas puntuaciones tanto en Ansiedad ante la agresión como en Ansiedad ante el fracaso escolar y las sanciones disciplinarias, puntuaron significativamente más elevado tanto en PSP como en PAO que sus iguales con bajas puntuaciones.

En este sentido, es necesario tener en cuenta que la Ansiedad es uno de los problemas emocionales con mayor trascendencia en la edad infanto-juvenil (Bessdo et al., 2009; Kessler et al., 2007). Si a ello le sumas que la etapa adolescente se trata de una población sensible debido a la búsqueda de su propia identidad y el vínculo con las variables perfeccionistas regidas por ese malestar personal (Aparicio-Flores et al., 2021), y de agotamiento y desgaste mental (Mahmoodi-Shahrebabaki, 2017), cabría tener en cuenta esta asociación como altamente vulnerable.

En cuanto al segundo objetivo, los resultados sugieren que a medida que aumenta la puntuación en PSP y PA0 aumenta la posibilidad de presentar altas puntuaciones tanto en Ansiedad ante la agresión como en Ansiedad ante el fracaso escolar y sanciones disciplinarias. Por una parte, cabe destacar estudios previos que muestran un vínculo entre el PSP y niveles de Ansiedad escolar y rechazo escolar (Essau et al., 2008; Stornelli et al., 2009). Por otra parte, es necesario tener en cuenta que, pese a no obtener datos anteriores de un vínculo directo entre el PAO y la Aansiedad escolar, sí se ha asociado con niveles de ansiedad en general (Essau et al., 2008; Stornelli et al., 2009).

Caben destacar diversas limitaciones del estudio. En primer lugar, este estudio no es generalizable a toda la población adolescente puesto que la muestra son estudiantes de Ecuador, por lo que un futuro estudio debería replicar el trabajo con muestra adolescente de otros países. Asimismo, pese a tener en cuenta que los instrumentos utilizados para la examinación de la Ansiedad escolar (García-Fernández et al., 2011) y el Perfeccionismo (Vicent et al. 2019; Vicent et al. 2020) son válidos y fiables, un futuro estudio debería emplear técnicas de observación 0 entrevistas cualitativas que permitan ampliar de forma más específica cuál es el origen de la ansiedad escolar y/o el perfeccionismo en cada caso de estudio. Del mismo modo, futuros estudios podrían examinar las diferencias entre sexo.

No obstante, y pese a observar estas limitaciones, los hallazgos percibidos manifiestan que tanto la Ansiedad ante la agresión como la Ansiedad ante el fracaso escolar y las sanciones disciplinarias es vinculado con el PSP y el PAO en grupos adolescentes ecuatorianos de entre 12 y 18 años de edad. Estos resultados ponen de manifiesto la necesidad de ampliar los estudios de Ansiedad escolar y Perfeccionismo en adolescentes de Ecuador con el fin de disminuir los riesgos asociados a ambas variables (Sung et al., 2006; Van Ameringen et al., 2003), teniendo en cuenta que cuando la ansiedad se da en la adolescencia y en la edad adulta, su desarrollo es de carácter gradual y se presenta con mayor intensidad y gravedad en la etapa adulta (Echeburúa-Odriozola, 2009; GarcíaFernández et al., 2008).

\section{CONCLUSIONES}

En conclusión, los resultados del presente trabajo contribuyen a ampliar el conocimiento científico en el campo de la psicología y la educación adolescente en el país de Ecuador. Del mismo modo, permiten continuar con estudios similares y abren la puerta a nuevos estudios que diseñen y pongan en práctica proyectos de intervención para prevenir o disminuir la Ansiedad escolar, así como el Perfeccionismo en adolescentes. 


\section{REFERENCIAS BIBLIOGRÁFICAS}

Aparicio-Flores, M.P., Esteve-Faubel, J.M., Vicent, M., Gonzálvez, C., Sanmartín, R., y García-Fernández, J.M. (2021). Dispositional empathy and emotional intelligence in terms of Perfectionistic Automatic Thoughts. The Spanish Journal of Psychology, 24, 1-10. https://doi.org/10.1017/SJP.2020.57

Aparicio-Flores, M.P., Vicent, M., Sanmartín, R., Gonzálvez, C., Freire-Andino, R.O., y García-Fernández, J.M. (2020). Psychometric properties of the Perfectionism Cognitions Inventory in Ecuador. International Journal of Environmental Research and Public Health, 17(16), 1-12. https://doi.org/10.3390/ijerph17165834

Babapour, K. J., Esmaeilpour, K. y Saeedi, D.S. (2015). The role of perfectionism in predicting feeling of cognitive, physical and social fatigue. Journal of Psychology, 19 (2), 163-174.

Batxer, A.J., Scott, J.M., Vos, T., y Whiteford, H.A. (2013). Global prevalence of anxiety disorders: a systematic review and meta-regression. Psychological medicine, 43(5), 897-910. https://doi.org/10.1017/S003329171200147X

Beesdo, K., Knappe, S., y Pine, D.S. (2009). Anxiety and anxiety disorders in children and adolescents: developmental issues and implications for DSM-V. The Psychiatric clinics of North America, 32(3), 483-524. https://doi.org/10.1016/j.psc.2009.06.002

Dadds, M.R., Holland, D.E., Laurens, K.R., Mullins, M., Barrett, P.M., y Spence, S.H. (1999). Early intervention and prevention of anxiety disorders in children: results at 2-year follow-up. Journal of consulting and clinical psychology, 67(1), 145-150. https://doi.org/10.1037//0022-006x.67.1.145

Echeburúa-Odriozola, E. (2009). Trastornos de ansiedad en la infancia. Pirámide.

Espada, J.P., Griffin, K. W., Pereira, J.R., Orgilés, M. y Garcia-Fernandez, J.M. (2012). Component Analysis of a School-Based Substance Use Prevention Program in Spain: Contributions of Problem Solving and Social Skills Training Content. Prevention Science, 13, 86-95. D0I: 10.1007/S11121-011-0249-Y

Essau, C.A., Leung, P.W.L., Conradt, J., y Cheng, H. (2008). Anxiety symptoms in Chinese and German adolescents: their relationship with early learning experiences, perfectionism, and learning motivation. Depression and Anxiety, 25(9), 801-810. https://doi.org/10.1002/da.20334

Esteve-Faubel, J. M., Aparicio-Flores, M. P., Vicent, M., Gonzálvez, C., Sanmartín, R. y García-Fernández, J. M. (2020). Validation of Spanish version of the Perfectionism Cognitions Inventory: profiles of automatic perfectionism thoughts and their associations with social anxiety. Professional Psychology: Research and Practice, 51(3), 268-277. https://doi.org/10.1037/pro0000290

Flett, G.L., Hewitt, P.L., Besser, A., Su, C., Vaillancourt, T., Boucher, D., Munro, Y., Davidson, L.A., y Gale, 0. (2016). The child-adolescent perfectionism scale: development, psychometric properties, and associations with stress, distress, and psychiatric symptoms. Journal of Psychoeducational Assessment, 34(7), 634-652. https://doi.org/10.1177/0734282916651381

García-Fernández, J. M., Inglés, C. J., Martínez-Monteagudo, M. C., Marzo, J. C. y Estévez, E. (2011). Inventario de Ansiedad Escolar: validación en una muestra de estudiantes de Educación Secundaria. Psicothema, 23(2), 301-307.

García-Fernández, J.M., Inglés, C.J., Martínez-Monteagudo, M.C., y Redondo, J. (2008). Evaluación y tratamiento de la ansiedad escolar en la infancia y adolescencia. Psicología Conductual, 16(3), 413-437.

Kessler, R.C., Amminger, G.P., Aguilar-Gaxiola, S., Alonso, J., Lee, S., Bedirhan Ustün, T. (2007). Age of onset of mental disorders: a review of recent literature. Current opinion in psychiatry, 20(4), 359-364. https://doi.org/10.1097/YC0.0b013e32816ebc8c

In-Albon, T., y Schneider, S. (2007). Psychotherapy of childhood anxiety disorders: a meta-analysis. Psychotherapy and Psychosomatics, 76(1), 15-24. https://doi.org/10.1159/000096361

Mahmoodi-Shahrebabaki, M. (2017). The effect of perfectionism on burnout among English language teachers: the mediating role of anxiety. Teachers and teaching, 23 (1), 91-105. https://doi.org/10.1080/13540602.2016.1203776 
Mendez, F.X., Espada, J.P., Orgiles, M., Hidalgo, M.D. y Garcia-Fernandez, J. M. (2008). Psychometric properties and diagnostic ability of the separation anxiety scale for children (SASC). European Child \& Adolescent Psychiatry, 17, 365-372. D0I: 10.1007/S00787-008-0678-8

Méndez, F. X., Inglés, C. J., Hidalgo, M. D., García-Fernández, J. M. y Quiles, M. J. (2003). Los miedos en la infancia y la adolescencia: Un estudio descriptivo. Revista Electrónica de Motivación y Emoción, 6(13). Recuperado de: http://reme.uji.es/articulos/amxndf4650710102/texto.html.

Nagelkerke, N.J.D. (1991). A note on a general definition of the coefficient of determination. Biometrika, 78(3), 691-692.

Stornelli, D., Flett, G.L., y Hewitt, P.L. (2009). Perfectionism, achievement, and affect in children: a comparison of students from gifted, arts, and regular programs. Canadian Journal of School Psychology, 24(4), 267-283. https://doi.org/10.1177/0829573509342392

Steel, Z., Marnane, C., Iranpour, C., Chey, T., Jackson, J.W., Patel, V., y Silove, D. (2014). The global prevalence of commom mental disorders: a systematic review and meta-analysis 1980-2013. International Journal of Epidemiology, 43(2), 476-493. https://doi.org/10.1093/ije/dyu038

Sung, K.M., Puskar, K.R., y Sereika, S. (2006). Psychosocial factors and coping strategies of adolescents in a rural Pennsylvania high school. Public Health Nursing, 23(6), 523-530. https://doi.org/10.1111/j.15251446.2006.00589.x

Van Ameringen, M., Mancini, C., y Farvolden, P. (2003). The impact of anxiety disorders on educational achievement. Journal of anxiety disorders, 17(5), 561-571. https://doi.org/10.1016/s0887-6185(02)00228-1.

Vicent, M., Inglés, C.J., Gonzálvez, C., Sanmartín, R., Aparicio-Flores, M.P., y García-Fernández, J.M. (2019). Self-criticism, strivings and aggressive behavior Spanish children: the two sides of self-oriented perfectionism. The Spanish of Journal of Psychology, 22, 1-8. https://doi.org/10.1017/sjp.2019.29

Vicent, M., Inglés, C.J., Sanmartín, R., Gonzálvez, C., Delgado, B., y García-Fernández, J.M. (2019). Spanish validation of the Child and Adolescent Perfectionism Scale: factorial invariance and latent means differences across sex and age. Brain Sciences, 9(11), 1-18. https://doi.org/10.3390/brainsci9110310

Vicent, M., Inglés, C.J., Sanmartín, R., Gonzálvez, C., Jiménez-Ayala, C.E., y García-Fernández, J.M. (2020). Psychometric properties of the child and adolescent perfectionism scale in ecuadorian adolescents. Journal of Affective Disorders, 272, 176-182. https://doi.org.10.1016/j.jad.2020.04.036

Vicent, M., Rubio-Aparicio, M., Sanchez-Meca, J. y Gonzalvez, C. (2019). A reliability generalization meta-analysis of the child and adolescent perfectionism scale. Journal of Affective Disorders, 245, 533-544. https://doi.org/10.1016/j.jad.2018.11.049 